\title{
BMJ Open Perioperative intravenous lignocaine infusion for postoperative pain control in patients undergoing surgery of the spine: protocol for a systematic review and meta-analysis
}

Ana Licina (D) , ${ }^{1}$ Andrew Silvers ${ }^{2}$

To cite: Licina A, Silvers A. Perioperative intravenous lignocaine infusion for postoperative pain control in patients undergoing surgery of the spine: protocol for a systematic review and meta-analysis. BMJ Open 2020;10:e036908. doi:10.1136/ bmjopen-2020-036908

- Prepublication history and additional material for this paper are available online. To view these files, please visit the journal online (http://dx.doi. org/10.1136/bmjopen-2020036908).

Received 10 January 2020 Revised 04 September 2020 Accepted 09 September 2020

Check for updates

(C) Author(s) (or their employer(s)) 2020. Re-use permitted under CC BY-NC. No commercial re-use. See rights and permissions. Published by BMJ.

${ }^{1}$ Anaesthesia, Austin Health, Heidelberg, Victoria, Australia ${ }^{2}$ Anesthesia, Monash Health, Clayton, Victoria, Australia

Correspondence to

Dr Ana Licina;

analicina@hotmail.com

\section{ABSTRACT}

Introduction Intravenous lignocaine is an amide local anaesthetic known for its analgesic, antihyperalgesic and anti-inflammatory properties. Administration of intravenous lignocaine has been shown to enhance perioperative recovery parameters. This is the protocol for a systematic review which intends to summarise the evidence base for perioperative intravenous lignocaine administration in patients undergoing spinal surgery.

Methods and analysis Our primary outcomes include: postoperative pain scores at rest and movement at predefined early, intermediate and late time points and adverse events. Other outcomes of interest include perioperative opioid consumption, composite morbidity, surgical complications and hospital length of stay. We will include randomised controlled trials, which compared intravenous lignocaine infusion vs standard treatment for perioperative analgesia. We will search electronic databases from inception to present; MEDLINE, EMBASE and Cochrane Library (Cochrane Database of Systematic Reviews and CENTRAL). Two team members will independently screen all citations, full-text articles and abstract data. The individual study risk of bias will be appraised using the Cochrane risk of bias tool. We will obtain a risk ratio or mean difference (MD) from the intervention and control group event rates based on the nature of data. We will correct for the variable measurement tools by using the standardised MD (SMD). We will use a random-effects model to synthesise data. We will conduct five subgroup analysis: major versus minor surgery, emergency versus elective surgery, patients with chronic pain conditions versus patients without, duration of lignocaine infusion and adult versus paediatric. Confidence in cumulative evidence for will be classified according to the Grading of Recommendations, Assessment, Development and Evaluation system. We will construct summary of findings tables supported detailed evidence profile tables for predefined outcomes.

Ethics and dissemination Formal ethical approval is not required as primary data will not be collected. The results will be disseminated through a peer-reviewed publication.

PROSPERO registration number CRD420201963314

\section{Strengths and limitations of this study}

- First proposed systematic review on the effects of intravenous perioperative lignocaine in patients having spinal surgery on outcomes such as static and dynamic pains scores, adverse events, opioid consumption amounts and quality of recovery.

- Conduct of meta-regression in order to assess the effect of lignocaine as a continuous independent variable on the outcomes.

- Application of Grading of Recommendations, Assessment, Development and Evaluation methodology to assess the quality of evidence across outcomes, with presentation of summary of findings tables.

- Management of meta-bias in the review including searching for published protocols, contrasting the structure with that of published study findings and review of selective reporting of outcomes.

- We anticipate limitations with regard to the heterogeneity of the included studies in relation to the elective/emergency nature of the procedure, anatomical site of surgery, dosing strategy/timing of the lignocaine bolus and outcome measures.

\section{INTRODUCTION}

The disease burden of spinal conditions needing surgery is high across the world. ${ }^{1}$ Since 2004-2015, volume of elective lumbar fusion has increased by $62.3 \%$ in the USA. Neurosurgical procedures encompassing spinal surgery have been traditionally associated with high analgesic requirements and prolonged functional recovery. ${ }^{2}$ Major efforts are being conducted to improve perioperative care of patients undergoing spine surgery. ${ }^{3}$ Improvements of perioperative analgesic pathways in surgery of the spine include maximisation of non-opioid management strategies. Lignocaine (lidocaine) has been described as having analgesic, antihyperalgesic, anti-inflammatory, antithrombotic, 
antimicrobial and antitumour properties. ${ }^{4-6}$ Prior systematic reviews have evaluated the efficacy and safety of perioperative intravenous lignocaine on postoperative outcomes in heterogenous types of surgery. ${ }^{78}$ There has been a significant benefit of intravenous lignocaine infusion observed in patients undergoing abdominal surgery. ${ }^{89}$ Specific effectiveness of intravenous lignocaine as an analgesic and/or antihyperalgesic in spinal surgery has not been previously assessed in a systematic review and meta-analysis.

\section{Description of the condition}

Open spine surgeries involve extensive dissection of subcutaneous tissues, bones, muscle and ligaments. This contributes to a considerable degree of postoperative pain. Postoperative pain in conventional spinal surgery can last for an average of 3 days. ${ }^{10}{ }^{11}$ Pre-existing pain conditions complicate effective postoperative pain management in this patient group. Conventional narcotics are often used to manage the pre-existing pain conditions. Patients undergoing spinal surgery are at risk of poor perioperative pain management due to medication tolerance, peripheral and central sensitisation syndromes as well as proinflammatory states. ${ }^{101213}$

\section{Description of the intervention}

Lignocaine is the first amino amide-type short-acting local anaesthetic developed in 1948. It was initially used as an antiarrhythmic agent, belonging to Ia group. Intravenous lignocaine infusion may be considered as a useful adjunct in perioperative pain management. ${ }^{1415}$ Lignocaine can be administered by an intravenous infusion intraoperatively or postoperatively as a component of multimodal pain strategy. A commonly used regimen includes an initial bolus (1.5-2 $\mathrm{mg} / \mathrm{kg}$ intravenous), followed by an infusion (1.5 to $3 \mathrm{mg} / \mathrm{kg} /$ hour intravenous), which can be continued postoperatively at a lower dose.$^{15}$ The optimal dose and duration of therapy are poorly defined. Please see table 1 for summary of pharmacokinetic properties of lignocaine ${ }^{15}$. When administered as an intravenous infusion in a perioperative setting, lignocaine has been found to have analgesic properties at concentration levels $0.5-5$ $\mu \mathrm{g} / \mathrm{mL}^{15}$

Intravenous lignocaine has both peripheral and central actions. Mechanisms of action of lignocaine include the following: blockade of sodium channels, glycinergic action, blockade of N-methyl-D-aspartate (NMDA) receptors, reduction in substance $\mathrm{P}$ and others (table 2). ${ }^{16} 17$ The exact molecular mechanisms of lignocaine action in modifying both acute and chronic pain are still somewhat elusive. ${ }^{17}$ As an anti-inflammatory agent, lignocaine inhibits leucocyte activation and adhesion to the site of injury in both in vitro and in vivo models. ${ }^{18}$ Lignocaine protects cells from inflammation by blocking the priming of neutrophils and therefore inhibiting the release of superoxide anions and interleukin-1B (IL-1B). ${ }^{19}$

Lignocaine is thought to reduce pain and hyperalgesia in neuropathic pain states. ${ }^{20}$ Lignocaine may inhibit spontaneous impulse generation from injured peripheral nerves and dorsal root ganglions proximal to the injured fibres. ${ }^{21}{ }^{22}$ Lignocaine is thought to suppress poly-synaptic reflexes in the dorsal horn, hence resulting in decreased nociception. ${ }^{23}$ In the electrophysiological experiments, intravenous lignocaine inhibits the excitatory postsynaptic currents evoked by noxious pinch stimuli. ${ }^{23}$ Intravenous systemic lignocaine may exhibit a central mode of action. ${ }^{24}$

Lignocaine is thought to have a slight negative chronotropic effect on the heart. The compound exhibits a biphasic action on smooth muscle of peripheral blood vessels, with vasoconstriction at low concentrations and vasodilation at higher concentrations. ${ }^{25}$ Lignocaine is thought to accelerate the resolution of postoperative ileus. ${ }^{15}$ The mechanism of this activity is poorly understood. Lignocaine has an antithrombotic effect through

Table 1 Summary of pharmacokinetic parameters of lignocaine

\begin{tabular}{|c|c|}
\hline Parameter & Value \\
\hline Therapeutic plasma level & $1.4-6.0 \mu \mathrm{g} / \mathrm{mL}$ \\
\hline Toxic plasma level & $8-12 \mu \mathrm{g} / \mathrm{mL}$ \\
\hline Maximum infiltrative dose with epinephrine & $7 \mathrm{mg} / \mathrm{kg}$ \\
\hline Elimination half-life $(\mathrm{t} 1 / 2 \beta)$ & $80-110$ min in healthy adults, predominantly via the hepatic route \\
\hline Metabolism & $\begin{array}{l}\text { Approximately } 90 \% \text { of lidocaine is metabolised in the liver by oxidative de- } \\
\text { ethylation (dealkylation) to monoethylglycinexylidide (MEGX) }\end{array}$ \\
\hline Metabolites & $\begin{array}{l}\text { MEGX exhibits less potent pharmacological properties comparable to } \\
\text { those of lignocaine }\end{array}$ \\
\hline
\end{tabular}


Table 2 Receptor/lon channel action of intravenous lignocaine

\begin{tabular}{|c|c|}
\hline Receptor/mechanism & Action \\
\hline Voltage gated sodium channel (VGSC) & $\begin{array}{l}\text { Classic mechanism of action is through blockade of VGSC }{ }^{17} \text {; } \\
\text { Intravenous lignocaine may inhibit VGSC in peripheral nerves and/or dorsal root } \\
\text { ganglia }{ }^{23} \text {; }\end{array}$ \\
\hline Potassium channels & $\begin{array}{l}\text { Lignocaine effect on potassium channels is available through experimental evidence } \\
\text { in animal models }{ }^{60} \text {; }\end{array}$ \\
\hline Calcium channels & $\begin{array}{l}\text { Lignocaine effect on calcium channels is available through experimental evidence in } \\
\text { human animal models }{ }^{17}\end{array}$ \\
\hline NMDA receptor & Lignocaine inhibits activation of NMDA receptor in a dose dependent manner ${ }^{2361}$ \\
\hline $\begin{array}{l}\text { Gamma aminobutyric acid (GABA) - } \\
\text { mediated chloride currents }\end{array}$ & $\begin{array}{l}\text { Lignocaine potentiates GABA-mediated chloride currents by inhibiting GABA uptake } \\
\text { in animal models }{ }^{62} \text {; }\end{array}$ \\
\hline Opioid receptors & Hypothesis mode of action unclear; \\
\hline Purine receptors & Hypothesis mode of action unclear; \\
\hline
\end{tabular}

an effective antiplatelet aggregating compound. ${ }^{4}$ The most common adverse effect of intravenous lignocaine is central nervous system toxicity. ${ }^{4}$ Symptoms are dose dependent (table 3). ${ }^{152627}$ Patients with heart failure or those with significant liver impairment, in whom metabolism of lignocaine is reduced, are particularly at risk.

\section{How the intervention might work}

In patients undergoing lumbar surgery, postoperative increase in neuroinflammatory markers has been demonstrated. ${ }^{28}$ Lignocaine can reduce the release of mediators of inflammation such as IL-4, IL-6 and tumour necrosis factor alpha. ${ }^{49}$ Lignocaine may specifically mitigate the neuroinflammatory process in patients undergoing spinal surgery. Neuropathic pain after spinal surgery, otherwise nominated as failed back surgery syndrome, is a frequently observed troublesome disease entity. ${ }^{30}$ There are no known fully successful therapeutic modalities. ${ }^{31}$ There may be a role for administration of perioperative intravenous lignocaine in preventing or decreasing the frequency of the failed back surgery syndrome. ${ }^{32}$ Central sensitisation is a predictor of a poor life quality and increased length of stay following multilevel spinal surgery. ${ }^{33}$ Lignocaine has the potential to decrease central sensitisation through multiple receptor mechanisms. ${ }^{16}$ Numerous other clinically relevant outcomes may be influenced by intravenous administration of lignocaine. These include: wound healing, coagulation, postoperative cognitive dysfunction, paralytic ileus and lung protection. ${ }^{17}$

\section{Why is it important to do this review?}

Analgesic strategies in patients undergoing spinal surgery are best implemented through multimodal regimens. ${ }^{13} 33$ Perioperative intravenous lignocaine may be a strategic component of multifaceted approach to analgesia in patients undergoing spinal surgery. A number of studies and meta-analysis have explored the effectiveness of perioperative intravenous lignocaine infusion in heterogenous surgical procedures. ${ }^{8}$ Pooling the pertinent data on the use of lignocaine in patients undergoing spinal surgery would allow us to estimate the perioperative effects specifically in this patient population.

\section{Aims and objectives}

This systematic review aims to assess the effects (benefits and risks) of perioperative intravenous lignocaine infusion compared with placebo/no treatment on

\section{Table 3 Summary of adverse effects of lignocaine}

\section{System \\ Adverse effects}

Neurologic side effects

\section{Cardiovascular side effects-infrequent;} associated with rapid administration

\section{Respiratory}

Other ranges
- Tremor, insomnia or drowsiness, lightheadedness, dysarthria and slurred speech, ataxia, depression, agitation, change in sensorium, a change in personality, nystagmus, hallucinations, memory impairment and emotional lability

- Very high plasma concentrations can result in seizures

(note seizure threshold is lower in the presence of pathophysiological abnormalities such as hypercapnia)

- Sinus slowing, asystole, hypotension and shock.

Patients with underlying heart disease are most at risk ${ }^{6364}$

- Bronchodilation at subtoxic doses; respiratory depression occurs at toxic dose

- Methemoglobinemia may occur at doses exceeding $600 \mathrm{mg}^{65}$ 
postoperative pain and recovery in patients undergoing spinal surgical procedures.

\section{METHODS AND ANALYSIS}

The present review protocol is being reported in accordance with the reporting guidance the Preferred Reporting Items for Systematic Reviews and Meta-Analyses Protocols (PRISMA-P) statement (see PRISMA-P checklist in online supplemental additional file 1). ${ }^{35}$

\section{Eligibility criteria}

Types of studies

We will include randomised controlled trials (RCTs) that evaluated the effect of perioperative lignocaine versus standard) treatment on relevant clinical outcomes in patients undergoing surgery on the spine. We define standard treatment as any analgesic modality which excludes perioperative lignocaine infusion. We will include studies published in the English language; however, our literature search will not be restricted by language of publication. As such, we will exclude the non-English language studies during the screening phase. Studies will not be excluded based on the publication status or reported outcomes.

\section{Types of participants}

We will include all patients undergoing spinal surgical procedures (box 1). We will include patients undergoing spinal surgical procedure on any anatomical site including cervical, thoracic, lumbar, sacral or any combination of these. We will include patients undergoing both elective and emergency surgery.

\section{Types of interventions and comparators}

We will include all studies comparing the effect of continuous perioperative lignocaine infusion as applicable to spinal surgery (table 4). Our intervention arm will consist of intravenous lignocaine administration which commenced perioperatively or intraoperatively (with or without a bolus) and continued postoperatively up to 72 hours following the completion of surgery. Our control arm will consist of any treatment excluding the intravenous lignocaine infusion (standard treatment).

\section{Types of outcome measures}

We will deem eligible all studies, which have included at least one of the following outcome measures:

Pain score scales such as Visual Analogues Scale (VAS) or Numerical Rating Scale (NRS), adverse events, quality of recovery (QoR) outcomes, patient-reported outcomes or length of stay (detailed outcome measures provided under 'Outcomes and Prioritisation' subheading.)

Our review eligibility criteria have been summarised in table 4 .

\section{Information sources}

We will search the MEDLINE via Ovid SP; EMBASE via Ovid SP; and Cochrane Library (Cochrane Database of
Box 1 Spinal surgery groups-both major and minor surgery included in the review

Anterior cervical decompression and fusion.

Posterior cervical decompression/fusion.

Thoracic decompression and fusion.

Scoliosis correction.

Multilevel decompression and fusion.

Lumbar decompression and fusion.

Lumbar laminectomy.

Lumbar microdiscectomy.

Systematic Reviews and CENTRAL). We will scan the trials registry, ClinicalTrials.gov for ongoing and unpublished trials. We will search the grey literature through the following specific search engines: Google scholar, http://www.opengrey.eu and http://www.greynet.org/ opengreyrepository.html. ${ }^{36-38}$

\section{Search strategy}

We will identify RCTs through literature searching with systematic and sensitive search strategies as outlined in Chapter 6.4 of the Cochrane Handbook for Systematic reviews of Interventions. ${ }^{39}$ Our search strategy was identified in collaboration with an information specialist. We will not apply study restrictions according to the language or publication status. For the search strategy, we will combine keyword and subject heading combinations in the predetermined databases. ${ }^{40}$ The search strategy specific to MEDLINE is included in online supplemental appendix one, available online. We intend to perform similar searches, with keywords adapted to specific database dictionaries.

\section{STUDY RECORDS}

\section{Data management}

Study information will be stored and managed using Endnote X9 throughout the review process. Collected data will be stored securely in an electronic format. Data will be made available on reasonable request 6 months following publication of the systematic review.

\section{Selection process}

Two reviewers will independently screen titles and abstracts using an electronic screening form

(covidence web platform: http://www.COVIDENCE. org). We will include articles for full-text review unless both reviewers deem them irrelevant. We will resolve any disagreement on study eligibility by author consensus. Results of the data search will be presented in a PRISMA flow diagram indicating the number of studies retrieved, screened and excluded as per exclusion criteria.

\section{Data collection}

Teams of two reviewers will perform data extraction using a predefined data collection form. Data will be extracted from each study based on prespecified criteria (please see 
Table 4 Summary of eligibility criteria

\begin{tabular}{|c|c|}
\hline Study characteristics & Inclusion criteria \\
\hline Patient population & All patients undergoing spinal surgical procedures \\
\hline Intervention treatment & $\begin{array}{l}\text { Intravenous lignocaine infusion which commenced perioperatively or } \\
\text { intraoperatively (with or without a bolus) and continued postoperatively up to } \\
72 \text { hours following the completion of surgery }\end{array}$ \\
\hline Comparator & $\begin{array}{l}\text { Standard care (any therapeutic modality excluding intravenous lignocaine } \\
\text { infusion); }\end{array}$ \\
\hline Outcomes & $\begin{array}{l}\text { Primary outcome measures: } \\
\text { 1. Postoperative pain score at rest at 'early'(1-4 hours) } \\
\text { 'intermediate'( } 4-24 \text { hours) and 'late' ( } 24-72 \text { hours postopertive) time } \\
\text { points } \\
\text { 2. Postoperative pain score with movement at 'early'(1-4 hours), } \\
\text { 'intermediate'( } 4-24 \text { hours) and 'late' ( } 24-72 \text { hours postoperative) time } \\
\text { points; } \\
\text { 3. Adverse events (dichotomous outcomes: all-cause mortality, } \\
\text { arrhythmias, other heart rate disorders or any sign of lignocaine toxicity); } \\
\text { Secondary outcome measures } \\
\text { 4. Intraoperative opioid consumption } \\
\text { 5. Postoperative opioid consumption, in 'postanaesthesia care unit } \\
\text { (PACU)' and 'overall', (in mg morphine equivalents. } \\
\text { 6. Postoperative nausea and vomiting at early time points (dichotomous } \\
\text { in 'PACU' and 'overall'); } \\
\text { 7. Functional postoperative neuropsychological status scales } \\
\text { 8. Composite morbidity including pulmonary, cardiac and renal } \\
\text { complication rates; } \\
\text { 9. Surgical complication rates as defined (dichotomous; postoperative } \\
\text { infections, thromboembolism, wound breakdown at 'early', 'intermediate' } \\
\text { and 'late time points'); } \\
\text { 10. Hospital length of stay (measured in days) }\end{array}$ \\
\hline
\end{tabular}

\begin{tabular}{|c|c|c|}
\hline Study design & Randomised controlled trials & $\begin{array}{l}\text { Case reports } \\
\text { Observational studies (cohort studies, } \\
\text { case-control studies, cross-sectional } \\
\text { studies, case series) } \\
\text { Non-randomised trials } \\
\text { Systematic reviews, meta-analysis; }\end{array}$ \\
\hline Study setting & $\begin{array}{l}\text { Inpatient care (including patients whose condition requires admission to a } \\
\text { hospital same day discharge surgical) }\end{array}$ & $\begin{array}{l}\text { Outpatient clinics, medical and } \\
\text { non-surgical management of spinal } \\
\text { conditions }\end{array}$ \\
\hline
\end{tabular}

data items). Data extraction forms will be piloted initially on a small number of included studies. Subsequently, each of the included studies will be abstracted by two team members, independently. Potential conflicts will be resolved through discussion. Authors of primary publications will be contacted for data clarifications or missing outcome data, as necessary.

\section{Data items}

Information of interest will include the following (online supplemental file data collection form):

- Study characteristics: study design, year of publication, journal, year (or period) of study conduct, sample size, setting, and other fields to capture data relevant to the assessment of study methodological quality (see risk of bias assessment subsection).

- Participant characteristics: population sampled, age (eg, mean with SD, range) and gender (eg, percentage of female participants), type of spinal surgery.

Exclusion criteria

Patients undergoing non-surgical management of spinal conditions Trauma patients without surgery

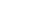




\section{Secondary outcome measures}

4. Intraoperative opioid consumption (remifentanil separated from other opiates due to unique short context sensitive half-life).

5. Postoperative opioid consumption, in 'postanaesthesia care unit (PACU)' and 'overall', (in mg morphine equivalents (MEQ).

6. Postoperative nausea and vomiting at early time points (dichotomous in 'PACU' and 'overall').

7. Functional postoperative neuropsychological status scales (eg, QoR score or Mini-Mental State Examination).

8. Composite morbidity including pulmonary, cardiac and renal complication rates.

9. Surgical complication rates as defined (dichotomous; postoperative infections, thromboembolism, wound breakdown at 'early', 'intermediate' and 'late time points').

10. Hospital length of stay (measured in days).

Time definition of early to late time points does involve some degree of arbitrariness. In line with other systematic reviews, we have used the common definition of early, intermediate and late as relating to the perioperative period. ${ }^{8}$ We defined early time point from 1 to 4 hours postsurgery. We defined the intermediate time point as 4-24 postoperatively. We defined the late time point as between 24 and 72 hours after surgery. We defined the 'overall time period' as the longest time point during which the patients were observed but within 72 hours of the completion of surgery. We have elected to evaluate morbidity as composite endpoint of cardiac, pulmonary and renal complications. We have chosen a composite endpoint for morbidity in order to increase statistical efficiency. We will use the weighted composites model to achieve the calculation of this outcome. ${ }^{41}$

\section{Risk of bias in individual studies}

Risk of bias in included studies will be assessed using the Cochrane 'Risk of Bias' tool. ${ }^{42}$ This tool is based on six key parameters. We will assess each component as being either low risk of bias, high risk of bias or unclear. We will include a 'Risk of bias' table as part of the 'Characteristics of included studies'. We will generate a 'Risk of bias summary', which will contain details of all of the judgements made. ${ }^{43}$ We plan to provide the details of the risk of bias assessment in a 'Critical Appraisal Form' supplied as an online appendix to the final manuscript.

\section{Random sequence generation}

We will consider the method of sequence generation as to ascertain its adequacy. We will consider the method as adequate if the sequence was generated by a random system. We will consider the sequence as inadequate if a non-random system was used.

\section{Allocation concealment}

We will consider the method of concealment as appropriate if steps were taken to ensure that the group assignment was not revealed to the participants, investigators or patient recruiters. We will consider the concealment to be inadequate if the allocation had been disclosed.

\section{Blinding of participants, personnel and outcome assessors}

We will allocate an appropriate risk of bias assessment according to the level of blinding of participants, study recruiters or investigators.

\section{Incomplete outcome data}

We will consider data provision as adequate if either there were no missing data or if reasons for missing data were adequately addressed (such as appropriate missing data imputation methods).

\section{Selective reporting}

We will seek prospectively registered study protocols with prespecified primary and secondary outcomes and ensure these are appropriately reported.

\section{Other sources of bias}

We will assess the studies for any other threats to validity such as early stopping of studies.

\section{Data synthesis}

Synthesis criteria under which study data will be quantitatively synthesised

We will assess the suitability to combine the data based on sufficient homogeneity and clinical judgement. Results will be evaluated based on a number of clinically relevant parameters including: commencement, timing, dosage and administration of intravenous lignocaine.

We will report statistical heterogeneity using the $\chi^{2}$ test statistic and the $\mathrm{I}^{2}$ statistic. Both will be calculated for each of the outcomes listed above. Statistical heterogeneity will be declared if the $\chi^{2}$ statistic has $\mathrm{p}<0.1$. We will use the following thresholds for interpretation of the $\mathrm{I}^{2}$ statistic in line with recommendations from Cochrane handbook ${ }^{40}$ :

- $0 \%-40 \%$ : might not be important.

- 30\%-60\%: may represent moderate heterogeneity.

- 50\%-90\%: may represent substantial heterogeneity.

- 75\%-100\%: considerable heterogeneity.

We will evaluate the importance of $\mathrm{I}^{2}$ depending on the magnitude and direction of effects as well as the strength of evidence for heterogeneity. ${ }^{40}$ If significant unexplained heterogeneity exists, or if there is an insufficient number of studies with appropriate outcome data for meta-analysis, we will describe data qualitatively.

\section{Measures of treatment effect}

For dichotomous outcomes, we will obtain a risk ratio (RR) from the intervention and control group event rates. For continuous data, we will obtain the mean difference (MD) from the difference between the intervention and control group mean values with associated SDs if all studies measured data on the same scale. The standardised MD (SMD) will be used when the studies assessed the same outcome but used variable measurement tools. For 
example, the VAS may be measured on a scale of $0-10 \mathrm{~cm}$ or $0-100 \mathrm{~mm}$. We will perform the back transformation of SMD values into absolute values on a scale of $0-10 \mathrm{~cm}$ (VAS) in order to assist with the clinical interpretation. All opioid quantities will be transformed into intravenous (MEQ mg) as described in the Defined Daily Dose index. $^{44}$

\section{Data synthesis-planned summary measures and methods of handling and combining data}

We will use Review Manager V.5.3 software for statistical analysis. We will use a random-effects model to analyse data. This will allow for the assessment of the true average effect of treatment across studies. We will be using inverse variance weighting summary of continuous outcomes and using Mantel-Haenszel methods for dichotomous outcomes. ${ }^{39}$ The outcomes will be presented with $95 \%$ CIs. This systematic review will compare patients receiving intravenous lignocaine versus patients receiving standard care (no intravenous lignocaine).

\section{Dealing with missing data}

If there are missing data (patient drop-outs or selective outcome reporting), we will contact the authors to obtain further information. If further information is not available, we will follow general recommendations for dealing with missing data in Cochrane Reviews ${ }^{40}$ :

- We will make explicit the assumptions of any methods used to address missing data.

- Appropriately assess the risk of bias due to missing outcome data in included studies.

- Perform sensitivity analyses to assess how sensitive results are to reasonable changes.

- Address the potential impact of missing data on the findings of the review in the Discussion section.

\section{Synthesis-subgroup analysis}

We will evaluate the magnitude of methodological and clinical heterogeneity. We will perform five subgroup analysis based on biological plausibility:

1. Major versus minor spine surgery. We will define major spine surgery as surgery incorporating more than a single-level spine surgery and/or lasting longer than 2 hours. ${ }^{45}$ Two level microdiscectomies will be considered as minor surgery.

2. Emergency versus elective spine surgery.

3. Patients with chronic pain conditions versus patients with no known chronic pain conditions as identified premorbidly.

4. Adult (patients older than 18) versus paediatric (patients younger than 18).

5. Perioperative lignocaine duration of infusion less than 24 hours, versus lignocaine duration of infusion for longer time period beyond surgery (greater than 24 hours but less than 72 hours).

We will perform subgroup analysis of these five groups calculating the $\mathrm{RR}$ or $\mathrm{MD}$, together with the corresponding CI, for each respective outcome. We will compare respective subgroup heterogeneity using the $\chi^{2}$ statistic. We will conduct meta-regression using Stata V.13 software in order to assess the effect of lignocaine as a continuous independent variable on the outcomes hypothesising that a higher cumulative dose of intravenous lignocaine may be more effective.

\section{Sensitivity analysis}

We will test the robustness of the effect estimates in sensitivity analysis for selection bias, blinding and attrition bias by excluding studies which we assessed as high or unclear risk of bias on their respective domains. If there are missing data from index studies, we aim to perform sensitivity analyses to assess how sensitive results are to reasonable changes. We aim to perform a meta-analysis using only low risk of bias studies in the evaluated domains in order to judge the robustness of summary statistics.

\section{Confidence in cumulative evidence}

Summary of findings' tables and Grading of Recommendations, Assessment, Development and Evaluation

We will use the Grading of Recommendations, Assessment, Development and Evaluation (GRADE) Handbook to guide the process of rating the quality of the body of evidence across individual outcomes including primary outcomes of pain score at rest and adverse events, and including all secondary outcomes. ${ }^{47}$ Quality of the body of evidence for the individual outcomes will be assessed according to study limitations, risk of bias imprecision, inconsistency of results, indirectness of evidence and publication bias. ${ }^{48}$ Evidence based on RCTs will be considered as high-quality unless confidence in the evidence is decreased due to study limitations, inconsistency of results, indirectness of evidence, imprecision and reporting biases. ${ }^{49}$ We will assess the quality of evidence for indirectness as adequate if the outcome data were based on direct comparisons of interest, on the population of interest, and on the outcome of interest. If assessed as inadequate, we will downgrade the inconsistency by one level. In order to judge for imprecision and inconsistency, we will examine the $95 \% \mathrm{CI}$ and the sample size. If the statistical test for funnel plot asymmetry suggests publication bias, we will downgrade the quality of evidence by one level.

We will use the GRADEpro software in order to generate the 'summary of findings (SoF)' table as provided by the guidance from the Cochrane handbook. ${ }^{39}$ We will construct an 'SoF' table for each primary and secondary outcome. We will support each 'SoF' table with a generation of a more detailed 'Evidence profile' table. The 'Evidence Profile' tables will provide greater detail than 'SoF' tables of both of the individual considerations feeding into the grading of certainty and of the results of the studies. ${ }^{50}$

\section{Meta-biases}

We will address publication bias by searching for published protocols of relevant studies. We will compare 
and contrast the structure of the published protocol with that of published study findings. Selective reporting of outcomes within studies will be reviewed by comparison with published protocols, review of study registration information on appropriate sources (eg, trial registration websites) ${ }^{40}$ We will construct a funnel plot of studies to assess for publication bias. We will assess the funnel plot for asymmetry. We will perform Egger's test to assess asymmetry of the funnel plot. ${ }^{51}$ The exhaustive search strategy has been designed in order to maximise study capture. Searches for relevant articles will be conducted in multiple databases and grey literature, minimising selection bias. Through prospective protocol registration and publication, we intend to promote transparency and replicability of this systematic review and meta-analysis.

\section{PATIENT AND PUBLIC INVOLVEMENT}

There was no patient involvement in the design of this protocol. We have considered the patient's needs in constructing this protocol by including patient reported outcome measures in the secondary outcomes of this study.

\section{ETHICS AND DISSEMINATION}

There will be no collection of individual patient data. Data collected in prior studies will be analysed in this systematic review. Therefore, ethics approval has been waived. We will disseminate the results of this systematic review and meta-analysis in a peer reviewed academic journal.

\section{DISCUSSION}

One of the primary underlying issues in spinal surgery is the morbidity of pre-existing pain and associated longterm analgesic requirements. ${ }^{52}$ Poorly controlled pain in the postoperative period can influence mobility and result in increased rate of complications of deep venous thrombosis, pulmonary embolism and pneumonia. ${ }^{53}$ Multimodal pain management has the potential to decrease postoperative pain, while reducing the total opioid consumption. ${ }^{53}$ Multimodal analgesia bundles have been incorporated into most care pathways of enhanced recovery in spinal surgery. ${ }^{1254}$ Intravenous lignocaine may have potential as a component of multimodal analgesia in patients undergoing surgery of the spine.

The main strength of our study stands in its design aimed at presenting a comprehensive evaluation of the effect of intravenous lignocaine infusion use during the perioperative period specifically in patients undergoing spinal surgery. By specifically targeting patients undergoing spinal surgery, we will be able to provide a clear description of both the potentially beneficial and harmful effects of this analgesic modality. Our study will provide an accurate and comprehensive understanding of the current evidence base on the effects of intravenous lignocaine on perioperative outcomes in this patient population.

Limitations of our study findings will relate to the methodological quality of the included RCTs. We will mitigate this by assessing the risk of bias at the study level through Cochrane Risk of Bias tool. Risk of bias will be assessed across outcomes as a part of the GRADE certainty of cumulative estimates tool. ${ }^{56}$ RCTs that are considered to have a low risk of bias or to be of high methodological quality could have some important design flaws not detected by methodological evaluations. Our planned outcomes may consist of diverse clinical endpoints, for example, variability in study definition of time points. We will deal with this limitation through careful evidence grading process, however, our study findings may be affected. The use of heterogeneous dosage regimens of intravenous lignocaine in some of the included studies might have an impact on the results observed and, thus, on the results of our meta-analysis. We anticipate underlying heterogeneity due to the variety of applicable spinal surgical procedures. Although we will be using a randomeffects model to synthesise data, clinical heterogeneity of the spinal procedures may affect the validity of the conclusion. Lignocaine administered intravenously has a narrow therapeutic index. ${ }^{15}$ Our review will not assess the extent of the therapeutic drug monitoring undertaken by individual studies. In the light of this, safety of intravenous lignocaine administration cannot be confirmed as we will not be assessing the plasma levels of lignocaine. ${ }^{5859}$

Intravenous lignocaine as an anaesthetic adjuvant may have the ability to improve the impact of multimodal analgesic regimens in spinal surgery. We expect our results will have implications for the assessment of the impact of perioperative intravenous lignocaine administration in patients undergoing spinal surgery.

Contributors AL reviewed the scientific literature and contributed to the original protocol. AS contributed to the original protocol.

Funding The authors have not declared a specific grant for this research from any funding agency in the public, commercial or not-for-profit sectors.

Competing interests None declared.

Patient consent for publication Not required.

Provenance and peer review Not commissioned; externally peer reviewed.

Supplemental material This content has been supplied by the author(s). It has not been vetted by BMJ Publishing Group Limited (BMJ) and may not have been peer-reviewed. Any opinions or recommendations discussed are solely those of the author(s) and are not endorsed by BMJ. BMJ disclaims all liability and responsibility arising from any reliance placed on the content. Where the content includes any translated material, BMJ does not warrant the accuracy and reliability of the translations (including but not limited to local regulations, clinical guidelines, terminology, drug names and drug dosages), and is not responsible for any error and/or omissions arising from translation and adaptation or otherwise.

Open access This is an open access article distributed in accordance with the Creative Commons Attribution Non Commercial (CC BY-NC 4.0) license, which permits others to distribute, remix, adapt, build upon this work non-commercially, and license their derivative works on different terms, provided the original work is properly cited, appropriate credit is given, any changes made indicated, and the use is non-commercial. See: http://creativecommons.org/licenses/by-nc/4.0/.

ORCID iD

Ana Licina http://orcid.org/0000-0001-8897-0156 


\section{REFERENCES}

1 Raciborski F, Gasik R, Kłak A. Disorders of the spine. A major health and social problem. Reumatologia 2016;54:196-200.

2 Murphy GS, Szokol JW. Intraoperative methadone in surgical patients: a review of clinical investigations. Anesthesiology 2019;131:678-92.

3 Wainwright TW, Immins T, Middleton RG. Enhanced recovery after surgery (ERAS) and its applicability for major spine surgery. Best Pract Res Clin Anaesthesiol 2016;30:91-102.

4 Beaussier M, Delbos A, Maurice-Szamburski A, et al. Perioperative use of intravenous lidocaine. Drugs 2018;78:1229-46.

5 Kastrup J, Petersen P, Dejgård A, et al. Intravenous lidocaine infusion--a new treatment of chronic painful diabetic neuropathy? Pain 1987;28:69-75.

6 Boas RA, Covino BG, Shahnarian A. Analgesic responses to i.v. lignocaine. Br J Anaesth 1982;54:501-5.

7 Vigneault L, Turgeon AF, Côté D, et al. Perioperative intravenous lidocaine infusion for postoperative pain control: a meta-analysis of randomized controlled trials. Can J Anaesth 2011;58:22-37.

8 Weibel S, Jelting Y, Pace NL, et al. Continuous intravenous perioperative lidocaine infusion for postoperative pain and recovery in adults. Cochrane Database Syst Rev 2018;6:CD009642.

9 Sun Y, Li T, Wang N, et al. Perioperative systemic lidocaine for postoperative analgesia and recovery after abdominal surgery: a meta-analysis of randomized controlled trials. Dis Colon Rectum 2012;55:1183-94.

10 Bajwa SJ, Haldar R. Pain management following spinal surgeries: an appraisal of the available options. J Craniovertebr Junction Spine 2015;6:105-10.

11 Debono B, Corniola MV, Pietton R, et al. Benefits of enhanced recovery after surgery for fusion in degenerative spine surgery: impact on outcome, length of stay, and patient satisfaction. Neurosurg Focus 2019;46:E6.

12 Elsarrag M, Soldozy S, Patel P, et al. Enhanced recovery after spine surgery: a systematic review. Neurosurg Focus 2019;46:E3.

13 Dunn LK, Durieux ME, Nemergut EC. Non-Opioid analgesics: novel approaches to perioperative analgesia for major spine surgery. Best Pract Res Clin Anaesthesiol 2016;30:79-89.

14 Weinberg L, Rachbuch C, Ting S, et al. A randomised controlled trial of peri-operative lidocaine infusions for open radical prostatectomy. Anaesthesia 2016;71:405-10.

15 Eipe N, Gupta S, Penning J. Intravenous lidocaine for acute pain: an evidence-based clinical update. BJA Educ 2016;16:292-8.

16 de Oliveira CMB, Issy AM, Sakata RK. Intraoperative intravenous lidocaine. Rev Bras Anestesiol 2010;60:325-33.

17 Hermanns H, Hollmann MW, Stevens MF, et al. Molecular mechanisms of action of systemic lidocaine in acute and chronic pain: a narrative review. Br J Anaesth 2019;123:335-49.

18 Hollmann MW, Gross A, Jelacin N, et al. Local anesthetic effects on priming and activation of human neutrophils. Anesthesiology 2001;95:113-22.

19 Caracas HCPM, Maciel JVB, Martins PMRES, et al. The use of lidocaine as an anti-inflammatory substance: a systematic review. $J$ Dent 2009;37:93-7.

20 Staud R, Weyl EE, Bartley E, et al. Analgesic and anti-hyperalgesic effects of muscle injections with lidocaine or saline in patients with fibromyalgia syndrome. Eur J Pain 2014;18:803-12.

21 Bräu ME, Dreimann M, Olschewski A, et al. Effect of drugs used for neuropathic pain management on tetrodotoxin-resistant $\mathrm{Na}(+)$ currents in rat sensory neurons. Anesthesiology 2001;94:137-44.

22 Tanelian DL, Victory RA. Sodium channel-blocking agents: their use in neuropathic pain conditions. Pain Forum 1995;4:75-80.

23 Kurabe M, Furue H, Kohno T. Intravenous administration of lidocaine directly acts on spinal dorsal horn and produces analgesic effect: an in vivo patch-clamp analysis. Sci Rep 2016;6:26253.

24 Koppert W, Ostermeier N, Sittl R, et al. Low-Dose lidocaine reduces secondary hyperalgesia by a central mode of action. Pain 2000;85:217-24.

25 Lieberman NA, Harris RS, Katz RI, et al. The effects of lidocaine on the electrical and mechanical activity of the heart. Am J Cardiol 1968;22:375-80.

26 Rademaker AW, Kellen J, Tam YK, et al. Character of adverse effects of prophylactic lidocaine in the coronary care unit. Clin Pharmacol Ther 1986;40:71-80.

27 Chopra MP, Thadani U, Portal RW, et al. Lignocaine therapy for ventricular ectopic activity after acute myocardial infarction: a double-blind trial. Br Med J 1971;3:668-70.

28 Repo JP, Häkkinen AH, Porkka T, et al. Increased interleukin-6 and C-reactive protein levels after instrumented lumbar spine fusion in older patients. J Orthop Surg 2019;27:230949901982640.
29 Garutti I, Rancan L, Simón C, et al. Intravenous lidocaine decreases tumor necrosis factor alpha expression both locally and systemically in pigs undergoing lung resection surgery. Anesth Analg 2014;119:815-28.

30 Cho JH, Lee JH, Song K-S, et al. Neuropathic pain after spinal surgery. Asian Spine J 2017;11:642-52.

31 Daniell JR, Osti OL. Failed back surgery syndrome: a review article. Asian Spine J 2018;12:372-9.

32 Ibrahim A, Aly M, Farrag W. Effect of intravenous lidocaine infusion on long-term postoperative pain after spinal fusion surgery. Medicine 2018;97:e0229-e.

33 Bajwa SJS, Haldar R. Pain management following spinal surgeries: an appraisal of the available options. J Craniovertebr Junction Spine 2015;6:105-10.

34 Bennett EE, Walsh KM, Thompson NR, et al. Central sensitization inventory as a predictor of worse quality of life measures and increased length of stay following spinal fusion. World Neurosurg 2017;104:594-600

35 Swartz MK. The PRISMA statement: a guideline for systematic reviews and meta-analyses. J Pediatr Health Care 2011;25:1-2.

36 Haddaway NR, Collins AM, Coughlin D, et al. The role of Google Scholar in evidence reviews and its applicability to grey literature searching. PLoS One 2015;10:e0138237.

37 Mahood Q, Van Eerd D, Irvin E. Searching for grey literature for systematic reviews: challenges and benefits. Res Synth Methods 2014;5:221-34

38 Paez A. Gray literature: an important resource in systematic reviews. J Evid Based Med 2017;10:233-40.

39 Brożek JL, Akl EA, Compalati E, et al. Grading quality of evidence and strength of recommendations in clinical practice guidelines Part 3 of 3 . The grade approach to developing recommendations. Allergy 2011;66:588-95.

40 Higgins JPT, Green S. Cochrane Handbook for Systematic Reviews of Interventions Version 5.1.0 [updated Mach 2011. The Cochrane Collaboration, 2011.

41 Duc AN, Wolbers M. Weighted analysis of composite endpoints with simultaneous inference for flexible weight constraints. Stat Med 2017;36:442-54.

42 Sterne JAC, Savović J, Page MJ, et al. Rob 2: a revised tool for assessing risk of bias in randomised trials. BMJ 2019;2:14898.

43 Guyatt GH, Oxman AD, Vist G, et al. GRADE guidelines: 4. Rating the quality of evidence--study limitations (risk of bias). J Clin Epidemiol $2011 ; 64: 407-15$.

44 Dukes MN. Drug utilization studies. methods and uses. Introduction. WHO Reg Publ Eur Ser 1993;45:1-4.

45 Polly DW, Chou D, Sembrano JN, et al. An analysis of decision making and treatment in thoracolumbar metastases. Spine 2009;34:S118-27.

46 Petrozza PH. Major spine surgery. Anesthesiol Clin North Am 2002;20:405-15.

47 Schünemann HBJ, Guyatt G, Oxman A. Grade Handbook for grading quality of evidence and strength of recommendations, 2013.

48 Balshem $\mathrm{H}$, Helfand $\mathrm{M}$, Schünemann $\mathrm{HJ}$, et al. Grade guidelines: 3 . rating the quality of evidence. J Clin Epidemiol 2011;64:401-6.

49 Guyatt GH, Oxman AD, Kunz R, et al. Going from evidence to recommendations. BMJ 2008;336:1049-51.

50 Guyatt G, Oxman AD, Akl EA, et al. Grade guidelines: 1. IntroductionGRADE evidence profiles and summary of findings tables. J Clin Epidemiol 2011;64:383-94.

51 Sterne JA, Egger M. Funnel plots for detecting bias in meta-analysis: guidelines on choice of axis. J Clin Epidemiol 2001;54:1046-55.

52 Deyo RA, Hallvik SE, Hildebran C, et al. Use of prescription opioids before and after an operation for chronic pain (lumbar fusion surgery). Pain 2018;159:1147-54.

53 Devin CJ, McGirt MJ. Best evidence in multimodal pain management in spine surgery and means of assessing postoperative pain and functional outcomes. J Clin Neurosci 2015;22:930-8.

54 Staartjes VE, de Wispelaere MP, Schröder ML. Improving recovery after elective degenerative spine surgery: 5 -year experience with an enhanced recovery after surgery (ERAS) protocol. Neurosurg Focus 2019;46:E7.

55 Muhly WT, Sankar WN, Ryan K, et al. Rapid recovery pathway after spinal fusion for idiopathic scoliosis. Pediatrics 2016;137. doi:10.1542/peds.2015-1568. [Epub ahead of print: 23 Mar 2016].

56 Sterne JAC, Savović J, Page MJ, et al. Rob 2: a revised tool for assessing risk of bias in randomised trials. BMJ 2019;366:14898.

57 Brozek JL, Akl EA, Alonso-Coello P, et al. Grading quality of evidence and strength of recommendations in clinical practice guidelines. Part 1 of 3 . An overview of the grade approach and grading quality of evidence about interventions. Allergy 2009:64:669-77. 
58 Greenwood E, Nimmo S, Paterson H, et al. Intravenous lidocaine infusion as a component of multimodal analgesia for colorectal surgery-measurement of plasma levels. Perioper Med 2019;8:1.

59 Wong BY, Hurwitz A. Simple method for maintaining serum lidocaine levels in the therapeutic range. Arch Intern Med 1985;145:1588-91.

60 Bräu ME, Nau C, Hempelmann G, et al. Local anesthetics potently block a potential insensitive potassium channel in myelinated nerve. J Gen Physiol 1995;105:485-505.

61 Hahnenkamp K, Durieux ME, Hahnenkamp A, et al. Local anaesthetics inhibit signalling of human NMDA receptors recombinantly expressed in Xenopus laevis oocytes: role of protein kinase C. Br J Anaesth 2006;96:77-87.
62 Hara K, Sata T. The effects of the local anesthetics lidocaine and procaine on glycine and gamma-aminobutyric acid receptors expressed in Xenopus oocytes. Anesth Analg 2007;104:1434-9.

63 Pfeifer HJ, Greenblatt DJ, Koch-Weser J. Clinical use and toxicity of intravenous lidocaine. A report from the Boston Collaborative drug surveillance program. Am Heart J 1976;92:168-73.

64 Schumacher RR, Lieberson AD, Childress RH, et al. Hemodynamic effects of lidocaine in patients with heart disease. Circulation 1968;37:965-72.

65 Gutenberg LL, Chen J-W, Trapp L. Methemoglobin levels in generally anesthetized pediatric dental patients receiving prilocaine versus lidocaine. Anesth Prog 2013;60:99-108. 